\section{POST DISCHARGE FOLLOW-UP FOR PATIENTS INFECTED OR COLONIZED WITH MULTIDRUG-RESISTANT ORGANISMS}

Roxanne Mora Taguas, Catu, Allan Mohideen, Juinarah Tana, Elma Lou Deyab, Ola Qudsi, Areej Yaseen, Muhammad AlAhmadi, Maher Al Saedi, Asim. Infection Prevention and Control Department, King Abdulaziz Medical City, Jeddah

\subsection{6/bmjoq-2019-PSF.26}

Background The spread of multidrug-resistant organisms (MDROs) among admitted patients is one of the major threats facing many hospitals in Saudi Arabia. These organisms include methicillin-resistant Staphylococcus aureus (MRSA), vancomycin- resistant enterococci (VRE), and certain gram-negative bacilli (GNB). There have been increasing challenges in providing and fully implementing specific infection control strategies for affected patients during their hospital stay. Current hospital infection control guidelines recommend single rooms for every MDRO colonized or infected patient. This should be continued while patients are in hospital and upon readmission unless patients are successfully cleared. Evidence of clearance from MDRO colonization is needed before patients are considered noninfectious. Although clearance of MDRO infected and colonized patients is successfully carried out during hospital stay, a major lack of such activity has been identified after patients are discharged from hospitals. The purpose of tracing patients post discharge is to ensure that they will be screened and re-swabbed during their outpatient appointments to assess whether they no longer require extra infection control measures, such as isolation precautions, during their subsequent admission, thereby reducing the need for single rooms.

Methods All patients discharged with MDROs were tracked by the assigned infection control practitioner (ICP). The ICP tracked the appointments of these patients. Notification and instruction for swabbing and rescreening were delivered to responsible nurses at the outpatient department (OPD) using the OPD notification forms.

Results Of 271 discharged patients with MDRO infection or colonization, 38 (14\%) patients were successfully cleared and deflagged from MDROs; 40 (14.7\%) were not given an OPD appointment; 19 (7\%) were not swabbed; 61 (22.5\%) have no doctor's order; 34 (12.5\%) were readmitted; 10 (3.6\%) were swabbed but still yielded positive results; 11 (4\%) were given very long appointment; and $9(3 \%)$ were transferred to another facility and/or home healthcare.

Conclusion This exercise proved to be very tasking for any ICP to undertake. In addition, multiple challenges have been identified which require administrative support, commitment, and participation of all healthcare workers to decrease demand on isolation beds and to reduce risk of MDRO transmission.

\section{MULTIFUNCTIONAL EVALUATION IN ELDERLY PATIENTS HOSPITALIZED FOR HEART DISEASE}

Eltayyeb Alameen, Amjad M Ahmed, lyad Farah, Mohammed Aziz. Cardiac Sciences, King Abdulaziz Cardiac Center, King Abdulaziz Medical City

10.1136/bmjoq-2019-PSF.27

Background Frail patients with cardiovascular disease have much higher frequencies of adverse events and complications, suggesting the need for more accurate functional stratification and careful evaluation of the risk/benefit ratio of invasive procedures. This project aimed to evaluate the prognostic impact of the Short Physical Performance Battery (SPPB) and handgrip test on the incidence of death and hospitalization for all causes in older patients hospitalized for heart disease.

Methods This prospective study included 283 patients aged 65 years or older, who between December 2015 and December 2017 were hospitalized for acute coronary syndrome, arrhythmias, or heart failure. Included patients were followed up after 1 year for the endpoint of all-cause mortality (ACM) and hospitalizations. All patients were evaluated for frailty using the handgrip test (using a dynamometer to measure the force of muscular contraction) and SPPB (to evaluate the functionality of the lower limbs). Chi-squared test and T-test were used to compare groups as appropriate. Univariate and multivariate logistic regression analysis was used to predict ACM.

Results Mean age was $72.8 \pm 6.5$ years, $70 \%$ of patients were male, and mean BMI was $29 \pm 5.3$. Acute coronary syndrome $51.5 \%$, length of stay $7.6 \pm 7.3$ days, diabetes $74 \%$, hypertension $79 \%$, and dyslipidemia $60.5 \%$. The total number of deaths was $27(9.5 \%)$ and total admissions $45 \%$. Charlson index in deceased participants was significantly higher than in alive participants $(p=0.039$, CI 0.04 1.33). Deceased participants were significantly frailer than those alive (SPPB $p=0.006$, CI -3.18 to -0.54 ). Admitted participants had higher Charlson index $(p=0.000$, CI 0.36 1.11) and were frailer than non-admitted participants $(p=0.003$, CI -1.9 to -0.38$)$ with statistical significance. Using univariate logistic regression only to predict ACM, Charlson index $\mathrm{p}=0.42$, odds ratio (OR) 1.25 (95\% CI 1.01-1.57); SPPB p=0.008, OR 0.854 (0.76-0.959). With multivariate analysis SPPB predicts ACM $(p=0.011$, CI 0.726-0.960).

Conclusion Among elderly patients (older than 65 years) hospitalized for heart disease (acute coronary syndrome, arrhythmia, heart failure) and after a year of follow up, the SPPB independently predicted all study outcomes (ACM, rehospitalization, and emergency room visit). Frailty evaluation can provide a valuable pre-discharge follow-up plan that might heavily impact patient care.

\section{INFECTION CONTROL MANAGEMENT OF CLOSTRIDIUM DIFFICILE INFECTION (CDI) IN AN INTENSIVE CARE SETTING AT KING ABDULAZIZ MEDICAL CITY/JEDDAH 2018}

Areej Qudsi, Roxanne Taguas, Ang Seokgor, Javid Bhutta, Fahad Alhameed, Asim Alsaedi Infection Prevention and Control Department Ministry of National Guard Hospital Affairs

\subsection{6/bmjoq-2019-PSF.28}

Background Clostridium difficile is a gram-positive, anerobic, toxin-producing bacillus that mainly causes diarrhea and colitis. A recent meta-analysis reported an overall rate of 3.54 per 10,000 patient-days per year for $C$ difficile infection (CDI). The highest incidence was observed in intensive care units (ICUs). In the USA, C difficile caused an estimated half a million infections and 29,000 deaths in 2012. More than $80 \%$ of these deaths occurred in individuals aged 65 years or older. The approximate cost of CDI treatment in the USA 
was US\$ 5.4-6.3 billion per year. For the countries in the Arabian Peninsula including Jordan, Kuwait, Qatar, Saudi Arabia, Egypt, and Lebanon, the prevalence rates range from $4.6 \%$ to $23.6 \%$ for CDI isolates. Saudi Arabia has the lowest rate $(4.6 \%)$ among these countries. A remarkable increase in nosocomial CDI cases in the adult ICU at King Abdulaziz Medical City was observed between 18 January and 11 March 2018.The aim of our study was to decrease the incidence of CDI in adult ICUs by implementing evidence-based interventions.

Methods Cluster investigations were done; a time, place, and person table created, brain storming to identify the possible risk factors was evoked by the investigative team, a root cause analysis/fishbone diagram was pulled, and corrective actions were formulated.

Clinicians: hand hygiene (soap and water), use of gloves, barrier precautions, prompt identification and early treatment of CDI cases were applied.

Environmental: proper cleaning, housekeeping protocol review, and types of disinfectants used were highlighted.

Surveillance: outcome surveillance of CDI cases was added to the patient safety annual report plan.

Administration: antibiotic stewardship program (ASP), managing staff shortage, and reinforcement of the guidelines were essential.

Results In the first quarter, the rate of CDI was 2.1 per 1000 patient-days, which dropped to 0.9, 0.4, and 0.4 per 1000 patient-days, respectively, in the second, third, and fourth quarters of 2018.

Conclusion There was a significant reduction in the number of CDI cases after timely and appropriate actions were taken. However, maintaining a zero rate was challenging. Continuous monitoring, ongoing data collection, and education were considered key to reducing CDI.

\section{IMPLEMENTATION OF AN ONCOLOGY ELECTRONIC REFERRAL SYSTEM TO IMPROVE ACCESS TO CARE FOR CHILDREN WITH CANCER (IMPACT): A QUALITY IMPROVEMENT INITIATIVE FROM A SINGLE CENTER}

Wasel Jastaniah, Taghreed Justinia, Saleh Maneea Allogmani, Riyadh Maneea Alloqmani, Basim Alsaywid, Amal Talal Alnakhli. College of Medicine, Umm Al Qura University, King Saud bin Abdulaziz University for Health Sciences, Ibin Senna

\subsection{6/bmjoq-2019-PSF.29}

Background Delayed access to cancer care has been associated with early childhood cancer death. Improving timely access to cancer care is the first important step in the cancer treatment journey. We implemented an electronic referral system (e-RS) at the Princess Noorah Oncology Center (PNOC) to improve timely access to cancer care. This study aimed to assess the impact of implementing an e-RS on timely access to cancer care.

Methods This was an observational retrospective analytical cross-sectional study of 399 pediatric oncology patients selected through a consecutive non-probability sampling technique to review the turnaround time (TAT) of children with cancer diagnosed 12 months before (manual referral system) and 12 months after implementation of the e-RS.

Results Of the 399 pediatric oncology patients diagnosed between January 2014 and December 2015, 59.91\% were male and $40.09 \%$ were female, with a median age for both sexes of 5.0 years (IQR 2.5-9.0 years). 96.3\% of the patients were Saudi and $3.7 \%$ were non-Saudi. The median processing time of the manual referral system was 1075 minutes (IQR 145-1498) while the median processing time for e-RS was 125 minutes (IQR 53-1013). By applying a Mann-Whitney test of significance, the TAT between the two systems was significant $(\mathrm{p}=0.0001)$.

Conclusion Implementing an e-RS improved referral TAT. As a result, pediatric oncology patients had improved timely access to cancer care at PNOC based on TAT standards. The impact of this improvement on outcomes will be reported separately.

\section{PREVALENCE OF HEPATITIS C VIRUS INFECTION AMONG A POPULATION TESTED AT KING ABDULAZIZ MEDICAL CITY, JEDDAH, IN 2018}

Mohammad Abdel Aziz, Fayssal Farahat, Majid AlThaqafy, John Ossenkopp, Lamiaa AlGhamdi, Asim AlSaedi. Infection Prevention and Control King Abdulaziz Medical City, Jeddah

\subsection{6/bmjoq-2019-PSF.30}

Background Hepatitis $\mathrm{C}$ virus (HCV) is one of the three major bloodborne infections. HCV infection has a devastating outcome, yet is a curable disease. The World Health Organization (WHO) targets elimination of the disease by 2030. This target cannot be achieved without screening and case detection. This retrospective study aimed to investigate the prevalence of HCV infection among people tested at King Abdulaziz Medical City, Jeddah (KAMC-J), during 2018.

Methods Results of the HCV antibody enzyme-linked immunosorbent assay (ELISA) tests conducted during 2018 at KAMC-J were obtained from the Electronic Medical System (BestCare). The HCV RNA test results were reviewed through the HCV surveillance database available in the Infection Prevention and Control Department. Data were also collected on demographic variables (age, sex, and nationality). Statistical analysis was done with IBM SPSS version 24.

Results A total of $5425 \mathrm{HCV}$ antibody tests were performed in 2018. More than half of the samples were for females (54.4\%, $\mathrm{n}=2953)$. The overall prevalence of positive $\mathrm{HCV}$ antibodies among the tested population was $1.5 \% \quad(n=82)$. Reactive HCV antibodies were higher among females $(1.6 \%$, $\mathrm{n}=46)$ than males $(1.5 \%, \mathrm{n}=36)$. The prevalence significantly increased with age from $0.3 \%(n=6)$ among people younger than 25 years up to $6.2 \%(n=42)$ among those older than 70 years. HCV positive antibody prevalence was significantly higher among Saudi $(1.8 \%, \mathrm{n}=79)$ than non-Saudi $(0.3 \%$, $\mathrm{n}=3$ ) populations. Of the 82 cases with positive $\mathrm{HCV}$ antibodies, $49(59.8 \%)$ cases were newly diagnosed, of which $30.6 \%(n=15)$ had reactive HCV RNA. Only two people were $\mathrm{HCV} / \mathrm{HBV}$ co-infected.

Conclusion This study demonstrates the importance of HCV screening of high-risk populations, including military personnel, and the need for early intervention in order to achieve the WHO target of eliminating the disease by 2030 . 